\title{
A Hybrid Hands-On And Computer Simulation Laboratory Activity For The Teaching Of Astronomical Parallax
}

\author{
Ned Ladd, Bucknell University, USA \\ Katharyn E. K. Nottis, Bucknell University, USA \\ Patricia Udomprasert, Center for Astrophysics, Harvard University \& Smithsonian, USA
}

\begin{abstract}
As computer-based visualization techniques are becoming more important across the landscape of astronomy education, this pre-test/post-test study using the Size, Scale, and Structure Concept Inventory (S3CI) looked at the impact of using a hybrid combination of hands-on and computer-based activities on the learning of five semesters of non-science majoring undergraduates learning about the concept of astronomical parallax. The hybrid laboratory activity comprises an outdoor component where students use the parallax method to determine the distances to nearby objects, and a computer visualization component using the American Astronomical Society's WorldWide Telescope astronomical visualization software. This activity was implemented as part of an undergraduate astronomy course for non-science majors. Based on an analysis of student responses, we conclude that this activity can help students understand the parallax method as applied in the astronomical realm. However, even after instruction, students had difficulty recognizing this method as the primary means for determining distances in astronomy.
\end{abstract}

Keywords: Parallax; Worldwide Telescope; Astronomy Laboratory Activities

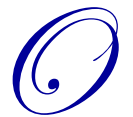

ur universe is inherently three dimensional, but because of its vast size and our essentially stationary location within it, we encounter the universe primarily as a two-dimensional "celestial sphere" rather than a rich volume filled with objects at a range of distances from our vantage point. Indeed, most astronomical measurements access directly only two of these three dimensions, and our knowledge of the third dimension (distance, or "depth") must be inferred from indirect techniques. The most fundamental of these indirect techniques is distance determination via astronomical parallax.

Parallax can be defined as the difference in the apparent position of a distant object when viewed from two different observing locations. Careful measurements of the change in apparent position and the separation between the two observation points can yield a distance to the object. Parallax is an everyday phenomenon that plays a role in our perception of distance through depth perception (via binocular vision), and in measurements of terrestrial distances via triangulation. As we move through our physical environment, viewing objects from multiple perspectives, we subconsciously determine their distances and construct a mental model of our three-dimensional surroundings. In the learning literature, this is referred to as "perspective taking" (e.g., Hegarty \& Waller, 2004; Höffler, 2010).

In the astronomical realm, multiple observational vantage points are afforded by the orbit of the Earth around the Sun. Astronomical observations conducted six months apart in time are physically separated by the diameter of the Earth's orbit, 2 Astronomical Units (A.U.; 1 A.U. $=1.5 \times 10^{11} \mathrm{~m}$ ). Though large by terrestrial standards, this distance is small compared to the distances to even nearby stars, and so the shift in the apparent positions of stars, typically 0.005 degrees or less, is too small to be discerned by eye. In fact, this shift was not successfully measured, even with telescopes, until 1838 (Bessel, 1838).

Learning about parallax in the astronomical context is difficult for a number of reasons. First, there is a lack of astronomy education in pre-college curricula. Previous research (e.g., Trumper, 2006; Sadler et al. 2010; Slater, Schleigh, \& Stork, 2015) has found that many students have little background knowledge of astronomical concepts 
when entering college. Without sufficient prior knowledge, it is difficult for students taking college astronomy classes to understand concepts such as parallax that cannot be directly observed within the timescale of a typical course. While it is possible to measure the parallax shift for some nearby stars with relatively inexpensive equipment, measuring the maximum shift requires observations separated by six months. Beyond the lack of background knowledge of basic astronomical concepts, the instructional methods used to teach parallax may also contribute to their difficulty. When parallax is taught, it is often in isolation from other relevant concepts (Fitzgerald, McKinnon, Danaia \& Woodward, 2011). For example, a commonly used approach for teaching parallax is the so-called "finger activity" where students hold up a finger at arm's length and then look at it with one eye and then the other, using the room as the setting. However, in most curricula, this is followed by an introduction of the parsec ${ }^{1}$ and the two concepts are never connected (Fitzgerald, et al. 2011).

In addition to parallax being taught in discrete lessons, Fitzgerald et al. (2011) has highlighted the problem of using a definitional approach when teaching difficult concepts, where students learn definitions without conceptual understanding. Other literature (e.g., Thomaz, Malaquias, Valente, \& Antunes, 1995) has indicated that these more traditional pedagogies can leave misconceptions, even after instruction. While there are varying views about conceptual change, recent thinking in this area indicates that it is not sudden but rather a slow process as other ideas are added to learners' understandings (Vosniadou, 2012; Vosniadou \& Skopeliti, 2014). Vosniadou and Skopeliti (2014) have explored this further, positing what they have labeled as a framework theory approach to conceptual change. Students initially construct a "naïve physics" based on observations and interaction with their environment but these ideas are resistant to concepts that are incompatible with their internal framework theory. Learners embrace them only when they can accommodate them incorrectly within their framework theory, or when their framework theory is successfully challenged as incorrect, inconsistent, or incomplete. Limited exposure to certain concepts and the use of instructional strategies where new information is not connected to previous concepts can lead to the continuance of students' misconceptions.

Like the atomic structure of molecules, or the internal structure of the Earth, parallax (and by extension the threedimensional structure of the universe) can only be taught via analogy and simulation. Though lacking a direct experiential component, these methods, particularly in combination, have been shown to be effective in teaching scientific phenomena that are not directly observable (e.g. Copolo \& Hounshell,1995; Gobert, 2000; McElhaney, Chang, Chiu \& Linn, 2015; Smetana \& Bell, 2012). Engaging with three-dimensional models can further build learners' understanding. Students' conceptual understandings can be influenced and changed through the process of such hands-on activities (Barnett, Keating, Barab \& Hay, 2013). This practice ultimately prepares them to engage more productively with the three-dimensional structures they will encounter in higher-level astronomy (Schneps et al. 2014).

Even when instructors use appropriate teaching methods, there is a lack of appropriate instructional materials or ones with inaccurate portrayals. In one study (Fitzgerald et al. 2011), when the researchers did a Google Image search using the term "Stellar Parallax," they found only $10 \%$ of the images were scientifically accurate and provided a full depiction of the concept. All of this can make the learning and teaching of parallax even more difficult.

Luckily, parallax is a scalable geometric effect, and therefore concrete terrestrial analogies for the astronomical phenomenon are easy to construct. Moreover, computer simulations allow students to engage with the parallax effect in a visualized astrophysical environment. In this paper, we present a preliminary assessment of the effectiveness of the combined laboratory activity, based on data from an initial implementation of this activity in an undergraduate astronomy course for non-major students.

\section{COMPUTER-BASED PSEUDO-3D VISUALIZATION USING THE WORLDWIDE TELESCOPE}

The American Astronomical Society's WorldWide Telescope (WWT) visualization software environment provides a framework for displaying and interrogating real astronomical data (Rosenfeld et al. 2018; Goodman et al. 2012; Wong, 2008). Stars, galaxies, and other celestial phenomena are accurately placed within WWT's three-dimensional universe

${ }^{1}$ A parsec is a distance corresponding to approximately 200,000 A.U.

Copyright by author(s); $\underline{\text { CC-BY }}$

The Clute Institute 
and are then displayed as viewed from the observer's perspective. Because the data can be placed in a threedimensional space (rather than only as points on a two-dimensional "sky"), the software can display the view from any location in the universe. This capability to change an observer's location means that a viewer's observation is no longer restricted to the surface of the Earth, and instead a student can "fly" through the universe, seeing how the arrangement of celestial objects changes with his/her position.

This change in the apparent positions of objects as a function of observer position is precisely the parallax effect, and so WWT is well-equipped to demonstrate this to students. WWT provides control over time and observer position, and so parallax effects that would be too slow and too small to observe in the real night sky can be made visually apparent in this virtual universe. In the real world, the maximum separation between observing positions is 2 A.U. (i.e., the diameter of the Earth's orbit), but with WWT, students can see how the arrangement of stars changes when viewed from locations separated by much larger distances. A larger separation between observing positions produces a more pronounced parallax effect -- one that is easily discernible to the naked eye.

The WWT software allows for the creation of "tours," scripted and guided interactions within the virtual universe that provide a framework for student investigation. Tours can be programmed to pause and prompt students to take control of the software to investigate a particular phenomenon or celestial object; in this way, students can be active learners within a framework that ensures an orderly and guided progression through a topic.

\section{CONTEXT OF THE STUDY}

This laboratory activity was designed and built to support instruction in a one semester introductory astronomy course (ASTR 102, "Stars, Galaxies, and Beyond") aimed at undergraduate non-science majors who need to fulfill a general education course requirement in laboratory science at a small, predominantly undergraduate university in Pennsylvania. The demographics of the students targeted for this study reflect the overall diversity of the institution: $50 \%$ female, $75 \%$ white, $7 \%$ Hispanic/Latina $6 \%$ Asian, and $4 \%$ black or African American. Nearly all students were of traditional college student age (18-22 years).

The course structure comprised three one-hour interactive lectures and one three-hour laboratory session each week. Total enrollment in the course ranged from 45 to 72 students; these students attended lecture together, and were split into multiple sections, each capped at 24 students, for the laboratory component. Students generally worked in pairs. The course was staffed by one faculty-level lecturer who often serves as a laboratory section instructor as well. Other laboratory sections were staffed by other faculty-level instructors, if needed. Laboratory session instructors were assisted by one or two undergraduate Teaching Assistants. Teaching Assistants are selected from a pool of students who have taken the course previously and excelled. Typically, they are not science majors.

The laboratory component is an amalgamation of hands-on, computer-mediated, and simulation instructional methodologies. It consists of twelve guided activities designed to provide students with opportunities to explore astronomical concepts. Activities involve hands-on exploration of concrete models (e.g., an illuminated sphere to investigate the phases of the Moon and Venus when observed from Earth), direct investigation of physical phenomena (e.g., visual spectroscopy of blackbody and spectral line emission), or manipulation of astronomical datasets (e.g., determination of the structure of the Milky Way galaxy from the spatial distribution of stars in the nighttime sky). Before each laboratory session, students complete a short online pre-lab reading that provides grounding and context for the laboratory activity and culminates with a short online quiz. The quiz allows for multiple trials, and students must answer all four quiz questions correctly before their assigned lab period.

During the laboratory session, students are provided with text-based guiding instructions; these instructions also contain diagnostic and interpretive questions designed to prompt students to reflect on the activity and develop a connection with relevant astronomical concepts. Students respond to these questions in writing during the laboratory session, and discuss their responses with peers and their instructor. The laboratory session concludes with a "Lab Quiz" comprising four short answer questions. These questions usually involve a short calculation based on the presented material, or an interpretation of data using concepts developed during the laboratory session. Student responses are graded, and their performance on this quiz accounts for a portion of their overall lab grade.

Copyright by author(s); $\underline{\text { CC-BY }}$ 


\section{The Parallax Lab Activity}

Our hybrid hands-on plus computer visualization activity was designed to fit within this course structure as a standalone three-hour $\mathrm{lab}^{2}$. Students are introduced to the concept of parallax, including the mathematical formalism, in the pre-lab reading. The scheduled lab period opens with a WWT tour that first describes the controls for the virtual environment and gives students an opportunity to practice. Since much of the lab involves changing observing position, it is essential that students understand how to manipulate these controls.

The tour then proceeds to display Orion, a well-known constellation in the winter sky. It demonstrates that the stars in this constellation are not all at equal distances from Earth by flying toward and through the constellation's stars. As the nearer stars pass by the observing screen, it becomes very clear that this constellation has its recognizable appearance only from the viewpoint of the Earth. As the viewpoint changes, the nearby stars appear to move more than the faraway ones, producing a jumbled arrangement of stars.

The tour then turns to the nearly universally-recognized asterism, the Big Dipper $^{3}$. Students are directed to compare the arrangement of these stars when viewed from two locations: the surface of the Earth, and then from a "friend's location" six parsecs from Earth. This distance is much larger than the size of our solar system, and comparable to the distances to some of the nearest stars, so the friend's location offers a very different perspective on the Big Dipper stars (Figure 1). Students are prompted to measure the angular shift in the directions to two of the Big Dipper stars due to this change in observing location, and to determine, based on these measurements, which of the Big Dipper stars is the most distant.

Emphasis is placed on the mechanics of the measurement process, including the need to measure angular shifts relative to a standard reference direction. In this exercise, the standard is provided by a star that is much farther from Earth than the Big Dipper stars. Since the parallax shift scales inversely with object distance, a very distant object is effectively stationary, and can be used to define a constant direction in space. Students measure angles directly on the computer screen, and the tour narration emphasizes the idea that physical distances measured on an image correspond to angular separations in the sky.

\footnotetext{
${ }^{2}$ Detailed notes and activity guides are available at the WWT Ambassadors website, http://wwtambassadors.org/bucknell-wwt-parallax-lab.

${ }^{3}$ The stars that form the Big Dipper are actually part of the constellation Ursa Major.
}

Copyright by author(s); $\underline{\mathrm{CC}-\mathrm{BY}}$ 
Figure 1. A screen shot of the WWT parallax tour. This image shows how the Big Dipper looks from a "friend's" location displaced six parsecs from Earth.

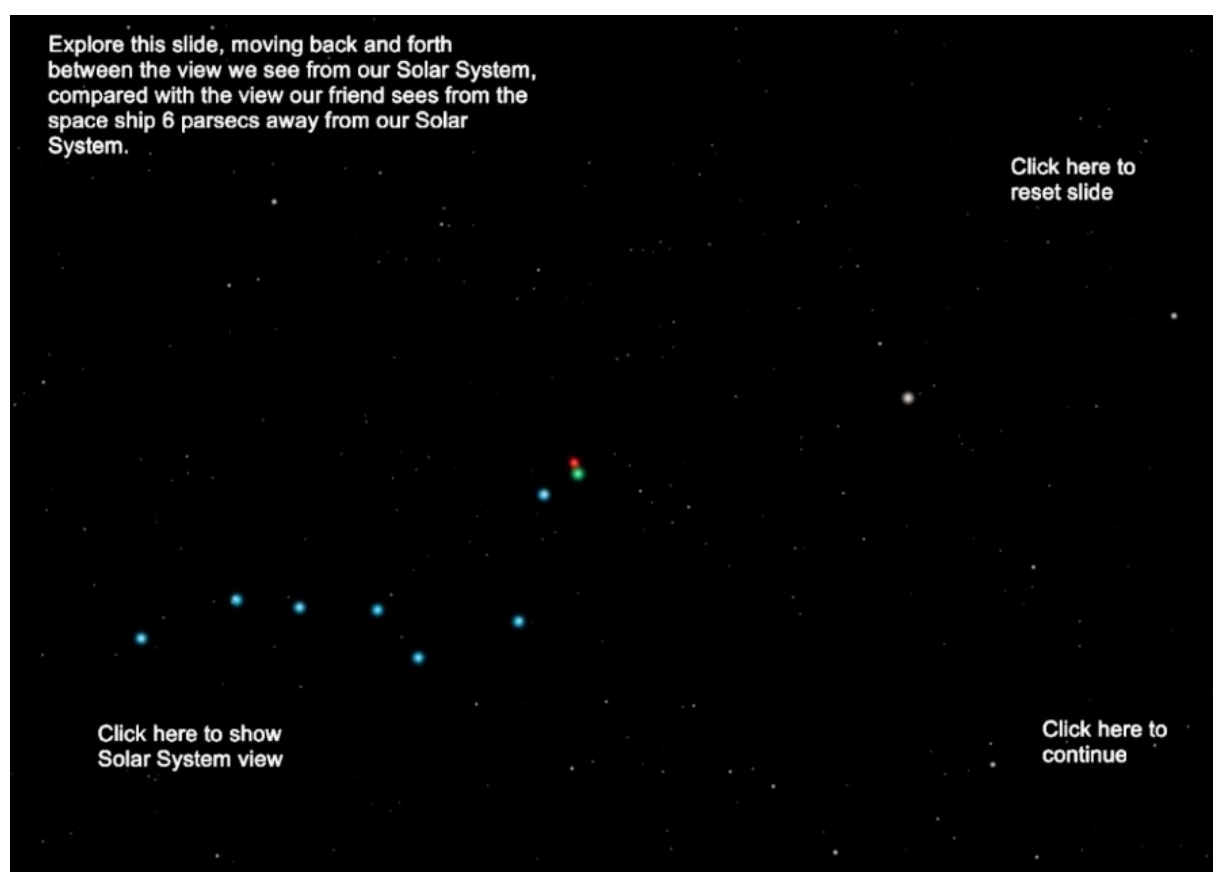

Throughout the measurement and evaluation process, the tour displays the view from both observing perspectives, as well as a displaced, "overhead" view that shows the geometry of the two locations and the Big Dipper stars. It is this last perspective that is most commonly presented in textbook diagrams and discussions of parallax, and a major goal of this section of the tour is to help students connect the in-situ observing perspectives with the overhead view.

At the conclusion of the WWT tour, students then move outdoors to measure parallax in the terrestrial environment. Here we construct a direct analogy for the Earth's orbit around the Sun with a 2-m diameter circle traced out in an open field (Figure 2). A lamppost approximately 40-m away serves as a nearby "star," and very distant objects visible along the horizon (e.g., church steeples, radio/cellphone towers) provide a standard reference direction (Figure 2). Students measure the angular separation between the direction to the nearby "star" and the distant standard using a surveyor's transit, which can measure angles with an accuracy of approximately 0.25 degrees. Students place the transit at several locations on the Earth's “orbit” and note how the angular separation changes with observing location. 
Figure 2. Students trace out a two-meter radius circle to mimic the Earth's orbit around the Sun (left). Then they measure the direction to nearby objects from several locations along the circle. Using a surveyor's transit, students measure the direction to a nearby lamp post, using the direction to a distant landmark as a reference (right).

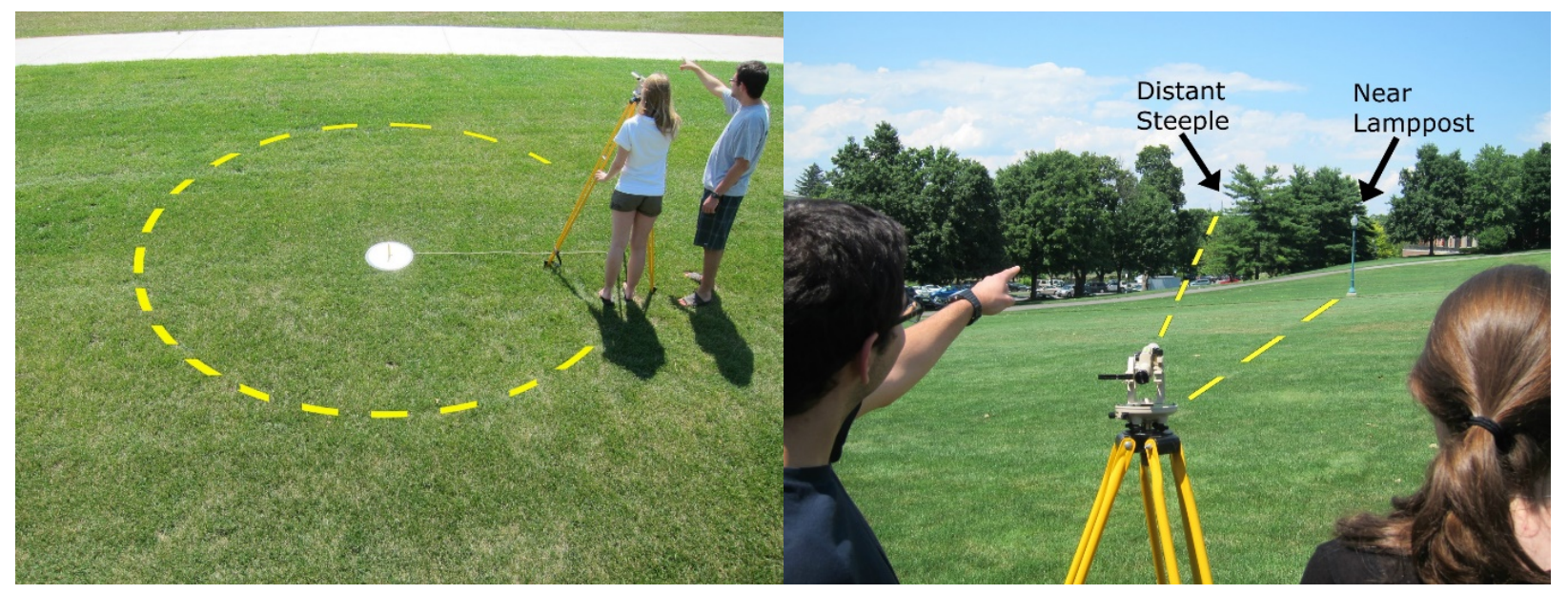

In this portion of the activity, they use the data collected and their knowledge of geometry to determine the distance to the nearby "star." The procedure is identical to that performed in real astronomical situation; however, the measurements can be made more quickly (i.e., no need to wait for the Earth to travel around the Sun), and the measured angles are larger because the nearby "star" is closer to the observer, relative to the size of the circular "orbit," than real stars are in the night sky. This calculation requires them to transfer their in-situ angle and distance measurements into an "overhead" diagram, on which they apply geometrical principles to determine the star distance.

The lab activity concludes with a Lab Quiz which requires students to perform a similar calculation with real astronomical data. They are also asked to explain aspects of the parallax measurement process, including how parallax angle scales with the distance to the object being measured, and why a distant reference object is necessary for this measurement.

\section{ASSESSMENT OF STUDENT LEARNING}

This lab activity was first introduced into the course environment in the fall semester of 2013, and it has been used in every subsequent instance of this course. Assessment of student learning was conducted during the first three offerings of the course, in the fall semesters of 2013, 2014, and 2015. Both the laboratory activity and our assessment instruments evolved over this period, as we learned more about student response to the material. For this paper, we present assessment results from the 2015 implementation. The following assessment tools will be discussed in this work:

- Size, Scale, and Structure Concept Inventory (S3CI) - One of the instruments used to assess students' learning was the S3CI (Nottis, Ladd, Goodman \& Udomprasert, 2015). This 24-item multiple choice diagnostic test was developed to assess undergraduates' understanding of astronomical size, scale and structure using well-known misconceptions as distracters. Some of the questions directly assess understanding of parallax in the terrestrial environment and in the astronomical realm. Although this evaluation tool was used in this exploratory study, the development of this instrument is ongoing, using what other researchers have labeled as an "iterative process" in its development (e.g., Petcovic \& Ruhf, 2008, p. 253; Wuttiprom, Sharma, Johnston, Chitaree \& Soankwan, 2009, p. 644). A more complete description, along with contact information for those interested in using this instrument, can be found at the WWT Ambassadors website (https://wwtambassadors.org/wwt-astro-101-labs). The data from individual questions discussed in this paper came from the 2015 administration of the $\mathrm{S} 3 \mathrm{CI}$ at the beginning and at the end of the semester to 38 and 27 students respectively in an introductory astronomy course. These pre-post responses for individual 
questions were then compared. The smaller sample size for this study restricted us to the use of nonparametric statistics, which do not have as much power and their parametric equivalents. While there were some trends toward statistically significant differences in the pre-/post- data, only a larger sample will resolve whether they truly are statistically significant.

- Post-Lab "Lab Quizzes" - Post-Lab "Lab Quizzes" were administered at the conclusion of each lab activity to determine if students understood the concept immediately after the lab activities. These quizzes consisted of four short answer questions. In one question, students are provided with input data for a parallax calculation, and are asked to use this method to determine the distance to a nearby star. The other three questions are more qualitative in nature, asking students to explain the aspects of the methodology, or to assess the limitations of the method.

\section{Results}

In this exploratory study, we examined how students responded to the lab activity, and tried to identify the factors that appear to limit their understanding of the parallax concept and its application in the astronomical environment. While the varying assessments do not provide a complete and conclusive view of the effectiveness of the parallax lab activity, student responses to individual questions show some evidence for learning success, and also illuminate persistent misconceptions. Below we discuss the student performance on some of these questions, supplemented by qualitative data from their written responses to quiz questions.

\section{Student Understanding of the Parallax Concept in the Terrestrial Environment}

Our data indicate that most students have a good intuitive understanding of the parallax concept in the terrestrial environment. Question \#20 of the S3CI (Figure 3) tests whether students can use their perspective-taking ability to determine the arrangement of three colored balls when viewed from two perspectives differing from the one shown. The question addresses directly the idea that one's view of objects at differing distances is affected by the position of the viewer. Student responses to this question in the 2015 administration of the S3CI are displayed in Figure 4. As can be seen from the figure, most students answered this question correctly in both the pre- and post-instruction administrations, with $65.79 \%$ answering correctly on the pre-test, and $77.78 \%$ answering correctly on the post-test. These results indicate that most students entered the course with an intuitive understanding of the observable consequences of the parallax effect, and that the laboratory activity appears to have increased the proportion of students who answer correctly. Interestingly, the most popular distractor, in both the pre- and post-instruction administrations, describes a view that is physically impossible to see from any perspective. It may be that students who chose this incorrect answer are not struggling with the concept of parallax, but instead with the basic perspectivetaking skills required to interpret the question. 
Figure 3. The image and text for Question \#20 of the S3CI

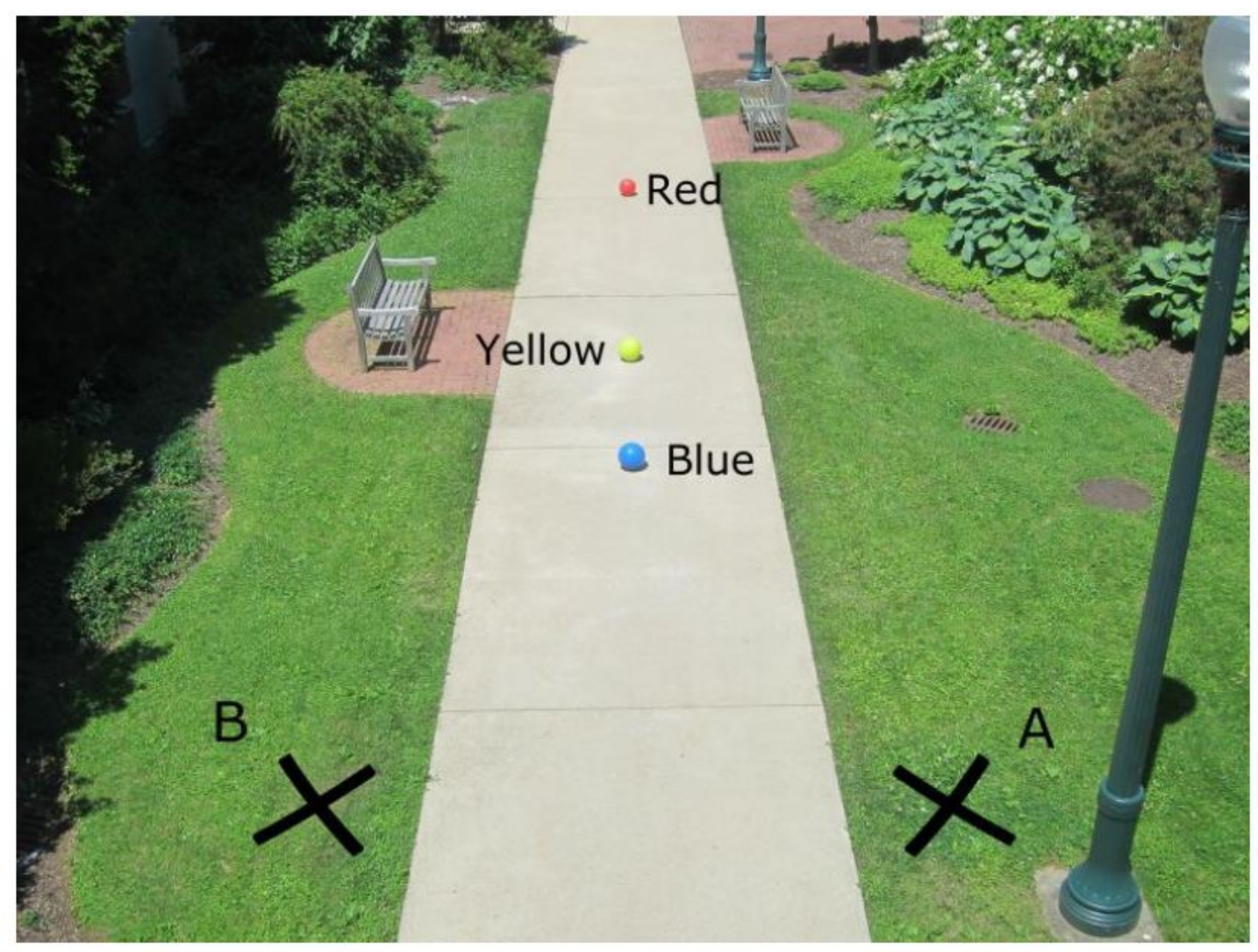

20. A blue ball, yellow ball, and red ball are located in a sidewalk as shown in the overhead photograph above. As you walk from position A to position B in the grass,
a. The blue ball appears to move from the right side of the yellow ball to the left side of the yellow ball, but remains to the right of the red ball.
b. The blue ball appears to move from the left side of the other balls, to the right side of the other balls.
c. The blue ball appears to move from the left side of the yellow ball to the right side of the yellow ball, but remains to the left of the red ball.
d. The relative order of the balls from left to right remains the same. 
Figure 4. Student responses to Question \#20 of the 2015 pre- and post-instruction administration of the S3CI.

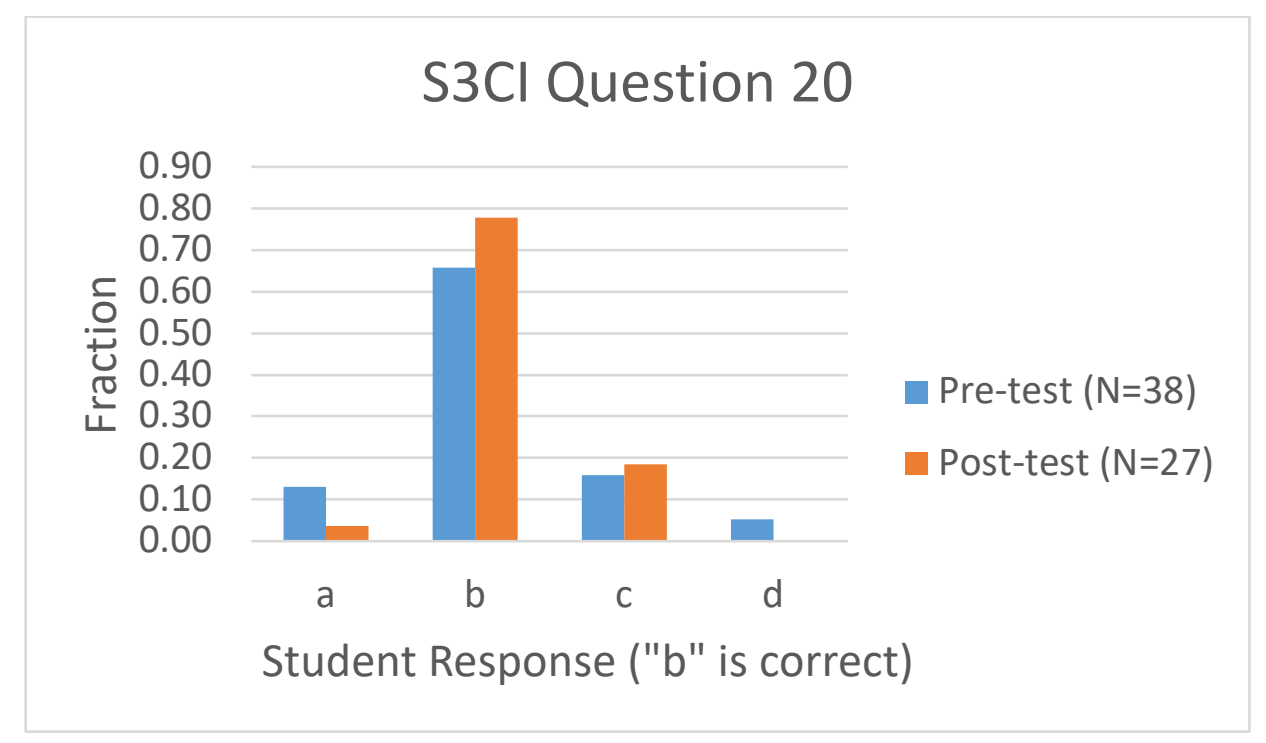

Our qualitative results support the conclusion that most students have a basic understanding of the observable consequences of the parallax effect in the terrestrial environment. After completing the outdoor activity described previously, students were asked on the Post-Lab quiz, "In this lab, you measured the distance to two lamp posts on campus. Why is your measurement of the distance to the nearer lamppost probably more accurate?" The majority of student responses to this question in the 2014 administration ( 25 of 38 students; $65.79 \%$ ) indicated an understanding that the shift in the apparent position of the nearer lamppost was larger, and therefore easier to measure accurately. A typical student response that was marked correct is as follows: "The nearer object appears to move more and thus is easier to measure accurately. More minute differences are harder to detect."

\section{Student Ability to Apply Parallax in the Astronomical Realm}

The WWT-enabled portion of the lab activity is designed to help students transfer their understanding of parallax in the terrestrial realm to the astronomical environment. This step is challenging, because although the basic geometrical relationships remain the same, the students' sensory experiences with parallax are quite different. The angular shifts of stars are much smaller than the shifts they see in the lamppost positions in the terrestrial case, and in most cases are not visually observable. Moreover, students do not consciously feel the change in observer position (due to the Earth's revolution around the Sun) as they do when moving around the circle in the outdoor component of the lab. We used the WWT software in an attempt to provide some visually apparent feedback by increasing the separation between observer positions, and thereby increasing the shift in apparent position.

Our Post-Lab quiz data show that students can apply the parallax concept in the astronomical environment. Most of them can correctly use the geometrical relationships to compute a star's distance, given an angular shift and the separation between observation points. Further, most students can explain the scaling relationships among the three relevant quantities: angular shift, separation between observation points, and object distance. For example, most students correctly respond that a larger separation between observing points will produce a larger angular shift for an object at a given distance.

\section{Student Understanding of the Parallax Concept to Infer Distances}

Despite their mechanical understanding, students still struggle with understanding the application and utility of the parallax method for determining distances in the astronomical environment. Question \#5 of the S3CI (Figure 5) illustrates their reluctance to rely on parallax as a method for determining distances. This question offers students 
several methods for determining the distances to nearby stars. Student responses to this question for the 2015 pre- and post-course administration of the S3CI are shown in Figure 6. In the pre-course administration, $42.10 \%$ of students respond correctly that distances are determined via parallax measurements. After instruction, the percentage answering correctly rises only slightly, to $48.15 \%$. In both the pre- and post-test data, the most attractive distractor involves using the brightness of stars to determine their distances. These students appear to discount the parallax method in favor of a more intuitive, yet incorrect method ${ }^{4}$.

Figure 5. The text for Question 5 on the S3CI

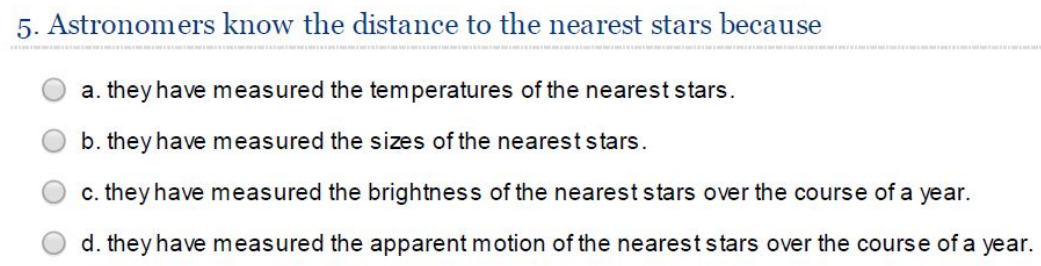

Figure 6. Student responses to Question \#5 of the 2015 pre- and post-instruction administration of the S3CI.

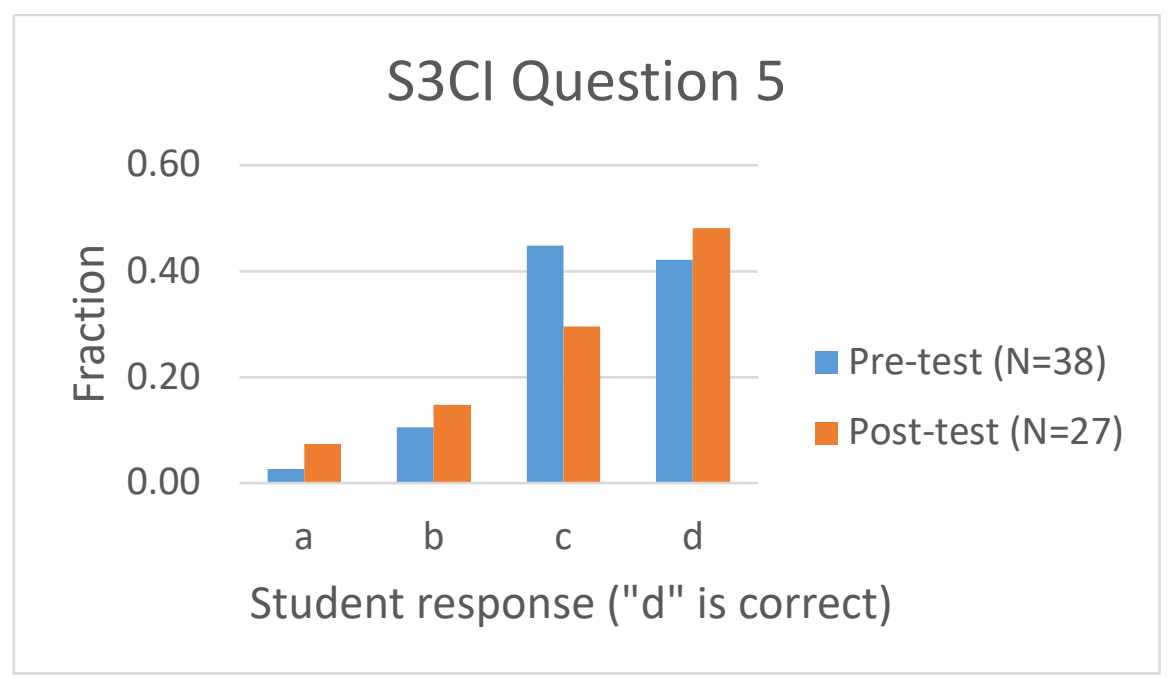

However, the evolution of their responses to S3CI Question \#6 (Figures $7 \&$ 8), however, is slightly more encouraging. They are presented with a situation where one star is observed to be brighter than another, and asked what they can say about the relative distances to the stars. In the pre-instruction administration, $65.79 \%$ correctly responded that the relative distances could not be determined from the given information, but $31.58 \%$ responded (incorrectly) that the brighter star was closer. After instruction, only $7.41 \%$ maintained this incorrect conclusion, while $85.18 \%$ answered correctly. Thus, students seem to be aware that brightness measurements alone are insufficient for the determination of distance, but based on their responses to Question \#5, they are not quite ready to embrace the parallax method as the correct method. Through their course experience, they may have begun to challenge their understanding of distance determination (i.e., brighter means closer), but have yet to integrate the new parallax method into their framework theory.

\footnotetext{
${ }^{4}$ Stars have a range of intrinsic luminosities, and so their apparent brightness is not a valid measure of their distance from an observer. 
Figure 7. The text for Question \#6 on The S3CI

6. Astronomers observe two stars in the night sky. One star is brighter than the other. From this information, they can conclude

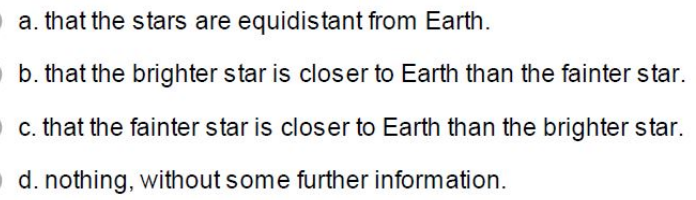

Figure 8. Student responses to Question \#6 of the 2015 pre- and post-instruction administration of the S3CI.

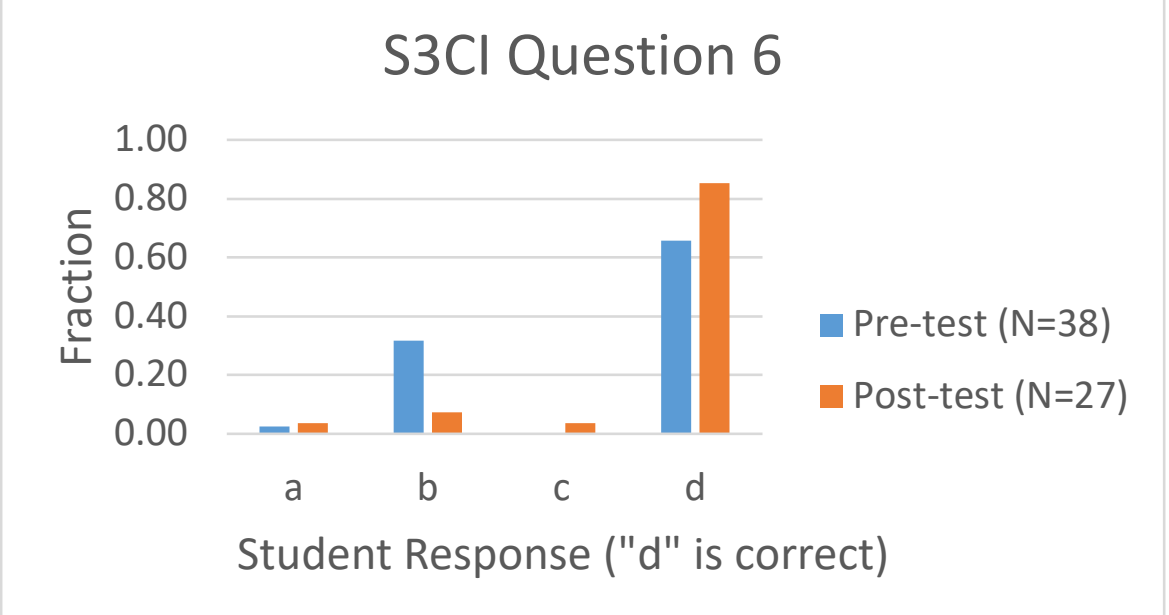

Vosniadou and Skopeliti (2014) identify this phase as one of "fragmentation." Students struggle with the inconsistency between new information and their existing framework theory, and as a result lose confidence in both. They note that "dissonance producing instructional interventions work only to the extent that the learner notices the discrepancy between their beliefs and the scientific information." (Vosniadou \& Skopeliti 2014, p. 1441). In this sense then, the parallax lab activity may be initiating the process of conceptual change, but for many of our students, further instruction is necessary for a complete transformation of their scientific understanding.

\section{CONCLUSIONS}

We have developed and tested a hybrid laboratory activity for the teaching of astronomical parallax to undergraduate students who are not majoring in science. Using hands-on lab activities and technology such as the World Wide Telescope (WWT) can aid students in better visualizing parallax and understanding how it works in regards to the stars (Ladd, Udomprasert, Nottis \& Goodman, 2016), and can help overcome some of the challenges in teaching and learning parallax. This lab activity appears to be helping students transfer their intuitive understanding of parallax to the astronomical context, so that they can correctly use this method to determine distances given appropriate inputs. However, despite their mechanical understanding, they do not readily recognize the parallax method as the primary means for distance determination in the universe.

\section{ACKNOWLEDGEMENTS}

This work was generously supported by the National Science Foundation through DUE-1140440. The authors would also like to acknowledge the work of Bucknell University students Emily Vislosky and Katelyn Kempf who assisted in analyzing the quantitative and qualitative data. 


\section{AUTHOR BIOGRAPHIES}

Ned Ladd is a Professor of Physics \& Astronomy at Bucknell University. His current research focuses on the use of computer visualization techniques in introductory astronomy laboratory instruction. E-mail: ladd@bucknell.edu

Katharyn Nottis is an Educational Psychologist and Professor Emerita in Education at Bucknell University. Her research has primarily concentrated on meaningful learning in science and engineering education from the perspective of Human Constructivism. She is committed to collaborative research projects, finding them a stimulating way to approach the complex problems in teaching and learning today. She has partnered with researchers in astronomy, chemistry, geology, seismology, and chemical and mechanical engineering. E-mail: knottis@bucknell.edu

Patricia Udomprasert is Project Director of the WorldWide Telescope Ambassadors Program at Harvard University. Her research focuses on the use of rich 3D visualizations to support spatial thinking and modeling of complex phenomena. E-mail: pudoomprasert@cfa.harvard.edu

\section{REFERENCES}

Barnett, M., Keating, T., Barab, S. A., \& Hay, K. E. (2013, April). Conceptual change through building three-dimensional virtual models. In Fourth International Conference of the Learning Sciences. Edited by: Fishman, B. and O'ConnorDivelbiss, S., (pp. 134-141).

Bessel, F.W. (1838). On the parallax of 61 cygni. Monthly Notices of the Royal Astronomical Society, 4, 152.

Copolo, C. F., \& Hounshell, P. B. (1995). Using three-dimensional models to teach molecular structures in high school chemistry. Journal of Science Education and Technology, 4, 295-305.

Fitzgerald, M. T., McKinnon, D. H., Danaia, L., \& Woodward, S. (2011). Using Smartphone camera technology to explore stellar parallax: Method results and reactions. Astronomy Education Review, 1.

Gobert, J. (2000). A typology of models for plate tectonics: Inferential power and barriers to understanding. International Journal of Science Education, 22, 937-977.

Goodman, A., Fay, J., Muench, A., Pepe, A., Udomprasert, P., \& Wong, C. (2012). WorldWide Telescope in Research and Education. Astronomical Data Analysis Software and Systems XXI. Proceedings of a Conference held at Marriott Rive Gauche Conference Center, Paris, France, 6-10 November, 2011, ed. P. Ballester, D. Egret, \& N. Lorente. ASP Conference Series: San Francisco, 267

Hegarty, M., \& Waller, D. (2004). A dissociation between mental rotation and perspective-taking spatial abilities. Intelligence, $32,175-191$.

Hoffler, T, (2010) Spatial Ability: Its influence on learning with visualizations - a meta-analytic review. Educational Psychology Review, 22, 245-269.

Ladd, E. F., Udomprasert, P., Nottis, K., \& Goodman, A. (2016). Building a three-dimensional universe from the classroom: Multiperspective visualization for non-science undergraduates.Proceedings of the 2016 International Conference on Education and New Developments, ed. M. Carmo, World Institute for Advanced Research and Science: Lisbon, 246250.

McElhaney, K. W., Chang, H., Chiu, J. L., \& Linn, M. C. (2015). Evidence for effective uses of dynamic visualisations in science curriculum materials. Studies in Science Education, 51, 49-85.

Nottis, K. E. K., Ladd, N., Goodman, A., \& Udomprasert, P. (2015). Initial development of a concept inventory to assess size, scale, and structure in introductory astronomy. US-China Education Review B, 5(11).

Petcovic, H. L., \& Ruhf, R. J. (2008). Geoscience conceptual knowledge of preservice elementary teachers: Results from the Geoscience Concept Inventory. Journal of Geoscience Education, 56(3), 251-260.

Rosenfield, P., Fay, J., Gilchrist, R. K., Cui, C., Weigel, A. D., Robitaille, T. ... Goodman, A. (2018). AAS WorldWide Telescope: seamless, cross-platform data visualization engine for astronomy research, education, and democratizing data. Astrophysical Journal Supplement Series, 236(1).

Sadler, P.M., Coyle, H., Miller, J.L., Cook-Smith, N., Dussault, M., \& Gould, R.R (2010). The astronomy and space concept inventory: Development and validation of assessment instruments aligned with K-12 National Journal of Astronomy \& Earth Sciences Education Science Standards. Astronomy Education Review, 8(010111-1).

Schneps, M.H., Ruel, J., Sonnert, G., Dussault, M., Griffin, M., \& Sadler, P.M. (2014). Conceptualizing astronomical scale: Virtual simulations on handheld tablet computers reverse misconceptions. Computers \& Education, 70, 269.

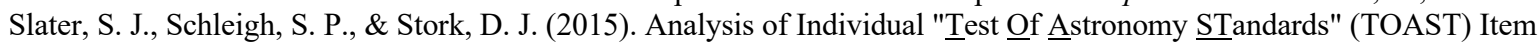
Responses. Journal of Astronomy \& Earth Sciences Education, 2(2), 89-108.

Thomaz, M. F., Malaquias, I. M., Valente, M. C., \& Antunes, M. J. (1995). An attempt to overcome alternative conceptions related to heat and temperature, Physics Education, 30, 19-26.

Copyright by author(s); $\underline{\text { CC-BY }}$ 
Trumper, R. (2006). Teaching future teachers basic astronomy concepts - seasonal changes - at a time of reform in science education. Journal of Research in Science Teaching, 43(9), 879-906.

Vosniadou, S. (2012). Reframing the classical approach to conceptual change: Preconceptions misconceptions and synthetic models. In Frazer, Tobin, McRobbie (Eds.), Second International Handbook of Science Education, 2, Springer International Handbooks of Education, 24, 119-130.

Vosniadou, S., \& Skopeliti, I. (2014). Conceptual change from the framework theory side of the fence. Science \& Education, 23, 1427-1445.

Wong, C. (2008). Contextual narrative as an information architecture for the WorldWide Telescope. Communicating Astronomy with the Public, 3, 22-25.

Wuttiprom, S., Sharma, M. D., Johnston, I. D., Chitaree, R., \& Soankwan, C. (2009). Development and use of a conceptual survey in introductory quantum physics. International Journal of Science Education, 31(5), 631-654. 
NOTES 